\title{
SOCIOLOGIE POLITIQUE DES PASSEURS
}

\section{ACTEURS DANS LA CIRCULATION DE SAVOIRS, DE NORMES ET DE POLITIQUES PUBLIQUES}

\section{Fabien Jobard, Bénédicte Laumond, Jérémy Geeraert, Irina Mützelburg, Ulrike Zeigermann}

La circulation des politiques publiques, de leur conception à leur instrumentation, est l'un des phénomènes les plus remarqués ces dernières années, sous l'effet bien sûr de la mondialisation des échanges et des interdépendances qui en résultent, de la prégnance des institutions internationales de financement ou de conseil, telles que le Fonds monétaire international, la Banque mondiale, l'Organisation de coopération et de développement économiques (OCDE), ou encore des efforts de convergence des États entre eux, des sommets internationaux aux ordres régionaux plus intégrés, tels que l'Association des nations d'Asie du Sud-Est (ASEAN) ou l'Union européenne. Un véritable «prisme circulatoire » marque ainsi aujourd'hui les recherches en science politique, prisme que l'on remarque d'abord au recours à un champ lexical emprunté à la géographie et à la communication : « échanges, transferts, diffusions, connexions, flux, réseaux ${ }^{1}$ », parfois encore à la géologie ou à la mécanique des fluides : « les fluctuations, les dilatations, les dénivellations ${ }^{2}$ ».

Les approches théoriques de ces phénomènes sont nombreuses et souvent plus anciennes que ce que l'on croit de prime abord: il suffit ici d'évoquer les critiques de l'impérialisme ou les modèles développementalistes ou encore la diffusion de modèles d'économie de marché ${ }^{3}$, d'ordo-libéralisme ou de gestion de l'entreprise entre l'Europe et les

1 Antoine Vauchez, «Le prisme circulatoire: retour sur un leitmotiv académique », Critique internationale, 59, 2013, p. 9-16, ici p. 9.

2 Pierre-Yves Saunier, «Circulations, connexions et espaces transnationaux », Genèses, 57, 2004, p. 110-126, ici p. 112. Sur les concepts aujourd'hui en usage, voir Elsa Tulmets, «Analyser l'exportation des normes au-delà de l'Union européenne : pour une approche éclectique », Politique européenne, 46, 2014, p. 832, voir en particulier p. 11 et 16. Ces concepts s'appuient souvent sur la construction de catégories et d'idéauxtypes - tels que l'imposition, l'harmonisation, la concurrence, la communication transnationale (ou l'apprentissage, voir plus loin la notion de lesson drawing) et la solution commune à des problèmes communs, développés par Katharina Holzinger, Christoph Knill, «Causes and Conditions of Cross-National Policy Convergence », Journal of European Public Policy, 12 (5), 2005, p. 775-796.

${ }^{3}$ Archie B. Carroll, Kareem M. Shabana, «The Business Case for Corporate Social Responsibility : A 
États-Unis, qu'elles furent transportées dans les valises du Plan Marshall et du «Washington Consensus » ou d'autres caravanes (dans une littérature abondante, voir les travaux de Nicolas Guilhot sur la Fondation Rockefeller ${ }^{4}$ ou de Dani Rodrick sur la Banque mondiale ${ }^{5}$, ceux de Jean-Paul Gaudillière sur les sciences du vivant ${ }^{6}$, ceux de Diane Stone sur 1'Open Society Institute ${ }^{7}$ ou ceux d'Yves Dezalay et Bryant Garth sur les juristes libéraux ${ }^{8}$ ). Certaines approches sont cependant plus neuves, comme en science politique dans le cadre de l'institutionnalisme sociologique ou des policy transfer studies $^{9}$, mais aussi des «mouvements sociaux transnationaux contemporains ${ }^{10}$ », en histoire dans le cadre de la world history ou de l'histoire globale ${ }^{11}$. Ces approches s'attachent à l'observation de divers

Review of Concepts, Research and Practice », International Journal of Management Reviews, 12 (1), 2010, p. 85-105 ; Oliver E. Williamson, « Opportunism and Its Critics », Managerial and Decision Economics, 14 (2), 1993, p. 97-107 ; Ulrike Zeigermann, Elsa Tulmets, « Policy Transfer in a Global Economy », dans E. Tulmets, Laure Delcour (dir.), Policy Transfer and Norm Circulation. Towards an Interdisciplinary and Comparative Approach, Londres, Routledge, 2019, p. 58-82.

${ }^{4}$ Nicolas Guilhot, «"The French Connection” : éléments pour une histoire des relations internationales en France », Revue française de science politique, 67 (1), février 2017, p. 43-67.

${ }^{5}$ Dani Rodrik, «Goodbye Washington Consensus, Hello Washington Confusion ? A Review of The World Bank's Economic Growth in The 1990s : Learning from a Decade of Reform », Journal of Economic Literature, 2006, 44 (4), p. 973-987.

6 Jean-Paul Gaudillière, «Mettre les savoirs en débat? Expertise biomédicale et mobilisations associatives aux États-Unis et en France », Politix, 57, 2002, p. 103-123.

7 Diane Stone, «Private Philanthropy or Policy Transfer? The Transnational Norms of The Open Society Institute", Policy \& Poltics, 38 (2), 2010.

${ }^{8}$ Yves Dezalay, Bryant Garth. «Droits de l'homme et philanthropie hégémonique », Actes de la recherche en sciences sociales, 121-122, 1998, p. 23-41.

${ }^{9}$ Magdaléna Hadjiisky, Leslie Alexander Pal, Christopher Walker, Public Policy Transfer : MicroDynamics and Macro Effects. New Horizons in Public Policy. Cheltenham, Northampton, Edward Elgar Publishing, 2017.

${ }^{10}$ Johanna Siméant, « La transnationalisation de l'action collective », dans Éric Agrikoliansky, Isabelle Sommier, Olivier Fillieule (dir.), Penser les mouvements sociaux. Conflits sociaux et contestations dans les sociétés contemporaines, Paris, La Découverte, 2010, p. 121-144.

11 Ajoutons à cela bien sûr que la transnationalisation des idées et des producteurs d'idées n'est pas seulement un objet des sciences sociales, mais un processus qui les traverse, comme le montrent Johan Heilbron, Laurent Jeanpierre, Nicolas Guilhot, «Vers une histoire transnationale des sciences sociales », Sociétés contemporaines, 73 (1), 2009, p. 121-145, ou les contributions réunies dans Gisèle Sapiro (dir.), L'espace intellectuel en Europe. De la formation des États-nations à la mondialisation, XIX ${ }^{e}-X X I^{e}$ siècle, Paris, La Découverte, 2009. 
objets empiriques, auxquels chacune accorde une importance plus ou moins forte selon son orientation: le savoir (les modèles, les théories, les connaissances) et ses modes de circulation, la structure institutionnelle caractérisant le territoire d'importation ou de réception et son environnement, les rapports de force ou de domination entre puissances exportatrice et importatrice. Ces modes d'analyse partagent un certain nombre de traits, mais aussi de risques communs, mis en évidence par de nombreux travaux de synthèse publiés par exemple sur les transferts de politiques publiques ${ }^{12}$.

Nous proposons ici de centrer l'analyse des transferts sur ses acteurs ou plus précisément sur une catégorie particulière d'acteurs, les passeurs, que nous définirons comme des acteurs individuels qui sont intermédiaires dans le processus de transfert d'une norme ou de pratiques, mais aussi producteurs ou récepteurs de ces normes ou pratiques. Ces acteurs se distinguent donc en ce qu'ils cumulent deux positions: producteurs et diffuseurs ou diffuseurs et récepteurs. Ce double positionnement distingue les passeurs des simples acteurs (au sens, par exemple, de policy makers) ou des courtiers : les premiers sont situés à la production des normes et des politiques publiques, les seconds à l'intermédiation.

Ce dossier contribue aux recherches sur les transferts et la circulation grâce à la notion de passeur. Après avoir introduit plus exactement la notion puis montré quelques avantages comparatifs, nous présenterons les textes qui, dans notre dossier, permettent de lui donner corps.

\section{L'analyse des transferts par le prisme des passeurs}

Lorsque l'on se représente les transferts, par exemple de politiques publiques, la tentation est forte de considérer que ceux-ci n'adviennent que par l'action d'acteurs spécialisés (durablement ou ponctuellement) dans l'intermédiation. Ce faisant, la sociologie du transfert se réduit à la sociologie de ce que sont et de ce que font les intermédiaires. Elle néglige alors les multiples activités sociales qui permettent le transfert, sans qu'elles fassent intervenir ces acteurs spécialisés et rétribués comme tels que sont les intermédiaires : des producteurs de normes, de pratiques, de politiques publiques peuvent avoir, à un moment ou

12 Voir parmi les plus marquants, en français, Thierry Delpeuch, «Comprendre la circulation internationale des solutions d'action publique : panorama des policy transfer studies », Critique internationale, 43 (2), 2009, p. 153-165 ; Laurence Dumoulin, Sabine Saurugger, «Les Policy Transfer Studies: analyse critique et perspectives », Critique internationale, 48 (3), 2010, p. 9-24. 
un autre, un intérêt particulier à exporter leur production et endossent alors un rôle de passeur ; des récepteurs ou destinataires de ces normes ou politiques peuvent eux aussi avoir un intérêt à importer (et, souvent, interpréter et transformer) ce qui est transféré. Sur les lieux de production ou sur les lieux de réception, des acteurs exportent ou importent, deviennent « passeurs », sans pour autant faire de cette activité un statut professionnel à part entière.

Nous envisageons donc de dépasser l'analyse des transferts depuis le seul statut d'intermédiaire en privilégiant la notion de rôle, notion qui permet de mieux faire la sociologie d'acteurs doublement positionnés (dans la production et la circulation, dans la circulation et la réception). Cette notion permet également de dépasser le biais élitiste qui caractérise une bonne partie de la littérature sur les transferts transnationaux, mais aussi de mieux envisager les risques d'une analyse trop strictement institutionnaliste.

Une définition par les rôles plutôt que par le statut : les passeurs

Afin d'englober l'ensemble des rôles que ces acteurs assurant le passage de savoirs peuvent endosser, nous nous saisissons de la notion de passeur parfois utilisée dans la littérature récente ${ }^{13}$, mais qui souffre encore d'un déficit définitionnel.

Un passeur est d'abord caractérisé en ce qu'il occupe simultanément plusieurs positions dans le processus circulatoire, ce qui permet de l'envisager comme intermédiaire et producteur ou récepteur d'un ensemble de savoirs. Parce qu'il ne s'agit pas d'un statut (par exemple professionnel), ce «passeur» peut être, comme l'évoque Thibaut Rioufreyt, un acteur «secondaire» et «moins visible» que le courtier investi du noble rôle de porter des savoirs, des normes ou des technologies nouvelles. Et parce que l'activité de transfert à laquelle il se livre n'est pas sa seule, ni même souvent sa principale activité, le passeur est requis de mettre en œuvre des aptitudes sociales mêlées : certes mettre en relation des entités, rendre audibles des discours ou des pratiques, mais aussi conserver (éventuellement) ou consolider la position qu'il occupe dans son champ propre.

Nous proposons donc une sociologie de ces activités hybrides qui consistent à transférer des normes tout en travaillant à leur production ou leur réception. Une vue centrée sur les seuls courtiers ne permet pas de saisir les multiples activités, souvent discrètes, des

${ }^{13}$ Geneviève Pagé, «La lente intégration du queer au féminisme québecois francophone : douze ans de résistance et le rôle de passeur des Panthères roses », Revue canadienne de science politique, 50 (2), 2017, p. 535-558; Thibaut Rioufreyt, «Les passeurs de la "Troisième Voie”. Intermédiaires et médiateurs dans la circulation transnationale des idées », Critique internationale, 59 (2), 2013, p. 33-46. 
acteurs situés à la production ou à la réception des normes et qui permettent le transfert de ces normes d'un lieu à l'autre. Ce faisant, notre approche des passeurs entretient une proximité notable avec celle présentée dans le programme sur «les courtiers du développement» proposé par Thomas Bierschenk et Jean-Pierre Olivier de Sardan ${ }^{14}$. Pour eux aussi, les courtiers sont des acteurs à la fois en position d'intermédiation et de réception des projets locaux de développement. Ces courtiers du développement acquièrent des compétences au fil de leurs pratiques et des opportunités, plus qu'ils ne disposent de capacités par leur statut ou leur position. Toutefois, ils peuvent endosser ce rôle de par la position sociale préalable qu'ils occupent, souvent de notables, voire de chefferies locales. Ces positions favorisent les échanges puis les liens durables avec les organismes de développement local, qui en retour s'appuient sur eux pour assurer la distribution de ressources nouvelles, dont les courtiers captent une partie. Ainsi, les courtiers du développement « deviennent des acteurs nouveaux qui s'ajoutent aux organisations intermédiaires et aux centres de pouvoirs locaux préexistants », acquièrent des positions qui leur permettent «d'orienter les politiques et les pratiques [...] dans le sens de leurs intérêts $»^{15}$. De leur côté, nos passeurs se distinguent de ces courtiers en ce qu'ils peuvent tout aussi bien se situer du côté des producteurs des normes, pas seulement des récepteurs. Ils s'en distinguent également en ce qu'ils, pour jouer ce rôle d'intermédiaire, ne sont pas nécessairement en position éminente (par exemple de notabilité, même seulement celle propre à «l'arène locale » qu'évoquent J.-P. Olivier de Sardan et T. Bierschenk). En effet, ils peuvent aussi, comme nous l'avons dit, occuper des positions tout à fait secondaires. De fait, ils peuvent devenir des «acteurs nouveaux», imposer des «enjeux nouveaux» dans les arènes locales, mais tout aussi bien ne pas s'engager dans un processus d'accumulation des ressources ainsi transférées (ou des ressources générées par le transfert), et quitter la scène locale une fois le transfert effectué ${ }^{16}$. Dans notre perspective, être passeur relève plus d'un rôle ponctuellement investi que d'un

${ }^{14}$ Jean-Pierre Olivier de Sardan et Thomas Bierschenk, «Les courtiers locaux du développement », Bulletin de l'APAD, 5, 1993.

15 Thomas Bierschenk, Jean-Pierre Chauveau, Jean-Pierre Olivier de Sardan, «Introduction. Les courtiers entre développement et État», dans Courtiers en développement. Les villages africains en quête de projets, Paris, Karthala, 2000, p. 6-12, ici p. xx.

${ }^{16}$ Les courtiers locaux du développement agissent dans un contexte où les biens en circulation sont bien plus des ressources matérielles que des normes abstraites, ce qui peut bien sûr susciter des intérêts plus immédiats, dans un contexte plus général, précisent Thomas Bierschenk et Jean-Pierre Olivier de Sardan, de « rente du développement ». 
statut hérité par la position dans le champ ou l'arène politique considérés ${ }^{17}$.

L'ambition de ce dossier est donc de mettre l'accent non plus sur les résultats ou les conditions de possibilité du passage d'une idée ou d'une norme d'un espace à un autre, mais plutôt de comprendre les dynamiques d'investissement du rôle de passeur, puis les implications de ce rôle sur la circulation des savoirs et sur les savoirs en circulation. À cette fin, nous présentons des passeurs impliqués de différentes façons dans la circulation de savoirs et de normes - que ce soit au sein d'une institution, entre institutions, à l'échelle locale, nationale, internationale ou transnationale. Car l'enjeu est bien ici de conjurer tout risque de stéréotypification du statut de passeur pour, à partir d'une sociologie de la diversité des contextes et des situations, ouvrir à la sociologie plus vaste et diversifiée du rôle de passeur. Par «rôle», nous entendons ici à la fois la prise de rôle et l'exercice du rôle. Ces notions permettent en effet, comme y appelait Jacques Lagroye, une appréhension des « comportements » tels qu'ils « résultent de l'état des rapports sociaux, et pas seulement des logiques propres » au secteur ou au champ considéré ${ }^{18}$. La notion de « rôle » porte mieux le caractère contingent et temporaire de l'activité de passeur et évite la réification et l'exclusivité qu'impose la notion de statut. Car nos passeurs ont bien un statut par ailleurs, comme celui de magistrat, celui d'employé d'une organisation non gouvernementale (ONG), de médecin, d'agent administratif, etc., qui leur permet ou les amène à endosser le rôle de passeur, mais qui ne les y oblige ni ne les y enferme ${ }^{19}$.

Les travaux menés jusqu'à présent sur les transferts de politiques publiques ${ }^{20}$ ou la diffusion des normes ${ }^{21}$ ont pour une grande part retracé et explicité les conditions de passage

${ }^{17}$ T. Bierschenk, J.-P. Chauveau, J.-P. Olivier de Sardan, « Introduction. Les courtiers... », op. cit.

${ }^{18}$ Jacques Lagroye, « Être du métier », Politix, 28, 1994, p. 5-15, ici p. 7 et 9.

${ }^{19}$ Ce point est un aspect important de ce qui distingue notre perspective de celle des anthropologues du politique Thomas Bierschenk et Jean-Pierre Olivier de Sardan, pour qui la «carrière » de courtier est une dimension importante de ce rôle social - même s'ils mettent en garde eux aussi contre toute réification des trajectoires des courtiers, évoquant du reste eux aussi des « occasionnels du courtage », lorsque cette activité est « un simple atout supplémentaire dans une vie professionnelle ou politique dont l'essentiel se joue ailleurs » (op. cit.).

${ }^{20}$ David Dolowitz, David Marsh, «Who Learns What from Whom : A Review of The Policy Transfer Literature », Political Studies, 44 (2), 1996, p. 343-357 ; David Dolowitz, David Marsh, «Learning from Abroad : The Role of Policy Transfer in Contemporary Policy-Making », Governance, 13 (1), 2000, p. 5-23.

21 Martha Finnemore, Kathryn Sikkink, «International Norm Dynamics and Political Change », International Organization, 52 (4), 1998, p. 887-917. 
des idées ou des référentiels d'un espace politique à un autre en insistant sur les arrangements et bricolages opérés alors. L'analyse questionne les spécificités des espaces institutionnels ou nationaux de départ et d'arrivée, mais, dans ce cadre, l'intérêt porté aux acteurs apparaît souvent secondaire.

Certains travaux sur les transferts s'intéressent certes aux acteurs, mais selon une approche mécaniciste inspirée par le paradigme rationaliste ${ }^{22}$, ce qui n'a pas manqué de susciter de nombreuses critiques ${ }^{23}$. Ces travaux supposent en effet que les transferts reposent sur l'action d'acteurs qui usent des transferts en jeu comme des investissements ou des coûts en vue de maximiser leurs ressources, leurs positions dans un champ donné, en bref : leur intérêt ${ }^{24}$. Prenant acte de ce biais simplificateur, les questionnements se sont alors multipliés et des recherches donnent à voir des transferts qui ne sont pas nécessairement le fruit des actions entreprises par des acteurs rationnels optimisant leurs intérêts, mais plutôt par des mécaniques d'interaction complexes qui ne se réduisent pas aux stratégies d'optimisation des acteurs engagés ${ }^{25}$. Mais, précisément, la mobilisation des acteurs dans l'analyse reste partielle : les acteurs ne sont étudiés qu'en tant qu'ils organisent ces circulations, comme si

${ }^{22}$ Colin Bennett, «What is Policy convergence and What Causes It ? , British Journal of Political Science, 21 (2), 1991, p. 215-233; Paul DiMaggio, Walter Powell(dir.), The New Institutionalism in Organizational Analysis, Chicago, University of Chicago Press, 1991 ; Erin Graham, Charles Shipan, Craig Volden, «The Diffusion of Policy Diffusion Research in Political Science », British Journal of Political Science, 43 (3), 2013, p. 673-701.

${ }^{23}$ Oliver James, Martin Lodge, « The Limitations of "Policy Transfer" and "Lesson Drawing” for Public Policy Research », Political Studies Review, 1 (2), 2003, p. 179-193 ; L. Dumoulin, S. Saurugger, «Les Policy transfer studies... », art. cité ; E. Tulmets, « Analyser l'exportation des normes... », art cité.

${ }^{24}$ Thomas Bierschenk, Jean-Pierre Chauveau et Jean-Pierre Olivier de Sardan relèvent que même l'anthropologie politique (fort éloignée des perspectives de transferts de politiques publiques) assigne au statut de courtier un prisme intéressiste. Chez un des premiers auteurs qui emploie le terme de courtier, Jeremy Boissevain, «Le courtier est un manipulateur professionnel de personnes et d'information qui produit de la communication pour le profit », dans Friends of Friends. Networks, Manipulators and Coalitions, New York, St. Martin's Press, 1974, p. 148. Notons cependant que ce profit n’est pas nécessairement monétaire, mais consiste le plus souvent à créer une obligation pour des transactions ultérieures.

25 Rodica Plugaru, «Le pouvoir des acteurs internationaux au sein des transferts: le rôle des organisations internationales et des entreprises privées dans la modernisation des hôpitaux en Ukraine et en Moldavie », Politique européenne, 46 (4), 2014, p. 60-85; Katerina Kesa, «Le partage de l'expérience balte envers les pays du voisinage orientale de l'Union européenne », Politique européenne, 46 (4), 2014 p. 86-116 ; Diane Stone, «Transnational Policy Entrepreneurs and The Cultivation of Influence : Individuals, Organizations and Their Networks », Globalizations, 16 (7), 2019, p. 1128-1144. 
cette activité leur était naturelle. Une sociologie de ces acteurs fait défaut, or elle permettrait de mettre en lumière les raisons pour lesquelles on transfère, les contraintes qui pèsent sur ceux qui s'engagent dans cette activité, tout aussi bien que les raisons qui font que l'on résiste au transfert, et les modalités de cette résistance.

Autre difficulté, la littérature sur ces questions estime trop souvent qu'existent différents types d'acteurs, qui peuvent être collectifs, individuels ou corporatistes, selon Fritz W. Scharpf ${ }^{26}$, ce qui dilue d'emblée une approche par les acteurs dans un ensemble de considérations où toute entité est susceptible de se voir honorée du qualificatif « d'acteur ». Individus, groupes sociaux et organisations structurées deviennent des «acteurs » ou, si le terme n'est pas employé, et pour ne citer que quelques exemples: des governance entrepreneurs, comme sous la plume de Philipp Pattberg ${ }^{27}$, ou des policy entrepreneurs, chez D. Stone ${ }^{28}$. Les intérêts sont alors indistinctement agrégés, tout comme les capacités d'action stratégiques, sans vraiment s'intéresser aux ressources nécessaires à l'action, lesquelles sont bien sûr différemment distribuées selon les individus et leurs places dans les champs sociaux et secteurs professionnels. Notre approche veut, à l'inverse, saisir la spécificité des acteurs porteurs de la circulation, en tant qu'individus socialement et professionnellement situés dans leurs champs respectifs, qui n'est pas celui de l'activité de transfert.

Les textes que nous présentons ici ont pour volonté commune d'approfondir les analyses menées sur les acteurs au centre de la circulation des savoirs en questionnant les rôles sociaux qu'ils investissent. Par «rôles sociaux » nous entendons l'activité sociale qu'ils déploient effectivement au cours de l'activité de transfert ou de passage, telle qu'elle résulte de leur socialisation, de leur position et des contraintes qui lui sont liées, enfin des attentes et des chances de succès des publics auxquels ils et elles destinent leur action ${ }^{29}$. La perspective

${ }^{26}$ Fritz W. Scharpf, Games Real Actors Play. Actor-Centered Institutionalism in Policy Research, Boulder, Westview Press, 1997.

27 «Je propose d'employer le terme «governance entrepreneur» pour qualifier ces individus, groupes d'individus ou institutions qui ont les caractéristiques évoquées », dans Philipp Pattberg, « The Emergence of Carbon Disclosure : Exploring The Role of Governance Entrepreneurs », Environment and Planning C. Politics and Space, 35 (8), 2017, p. 1343-1361, p. 1439 (traduction des auteurs).

${ }^{28}$ L'Open Society Institute n'agit pas seul dans ses interventions intellectuelles, mais le plus souvent en coalition avec des penseurs et militants partageant ses vues dans les secteurs du journalisme, de l'université ou d'autres professions intellectuelles », dans D. Stone, «Private Philanthropy or Policy Transfer... », art. cité (traduction des auteurs).

${ }^{29}$ Olivier Nay, «L’institutionnalisation de la région comme apprentissage des rôles : le cas des 
de recherche se déplace alors quelque peu, et ce qui devient central dans l'analyse des transferts et des circulations est bel et bien la prise de rôle éventuelle, c'est-à-dire la consistance ou au contraire la friabilité sociale de l'investissement dans l'acte de transférer, de passer, selon bien sûr les gains que l'on en escompte, mais aussi la position que l'on occupe, les chances de succès que l'on identifie, la conviction que l'on accorde à ce que l'on manipule alors.

Ce questionnement sur le transfert et la circulation devient une enquête sur la figure des passeurs, une sociologie de ce rôle social particulier. La diversité des situations et des objets retenus dans ce dossier permet ainsi d'échapper à la figure imposée de l'intermédiaire ou du courtier, de ne pas seulement étudier les rôles les plus éminents (comme les diplomates ou les fonctionnaires d'organismes transnationaux) ou les plus visibles (comme les «élites mondialisées »), pour comprendre ce que les transferts doivent à la multiplicité des rôles sociaux qui les permettent et que nous embrassons sous le terme plus large de « passeur ».

Dépasser une approche par les élites

Dans la perspective ouverte par de précédentes recherches ${ }^{30}$, notre dossier prend donc les acteurs du transfert, les passeurs, comme cibles de l'analyse. Faire la sociologie des passeurs permet également de mieux éprouver ce qui est en jeu dans les résistances opposées à certaines tentatives de transferts et de restaurer les dynamiques conflictuelles en jeu dans l'activité même de passer, de transférer, de faire circuler. Il s'agit pour nous de donner corps au dépassement souvent revendiqué de la dichotomie promoteurs/destinataires de normes ${ }^{31}$, mais aussi de la dichotomie opposant acteurs étatiques et non étatiques ${ }^{32}$. En bref, de sociologiser les relations de pouvoir que les transferts structurent, altèrent ou transforment ${ }^{33}$.

conseillers régionaux », Politix, 38, 1997, p. 18-46, ici p. 21.

30 Sophie Jacquot, Cornelia Woll (dir.), Les usages de l'Europe. Acteurs et transformations européennes, Paris, L'Harmattan, 2004 ; Olivier Nay, Andy Smith (dir.), Le gouvernement du compromis. Courtiers et généralistes dans l'action politique, Paris, Économica, 2002, voir p. 47-86 ; Patrick Hassenteufel, Jacques de Maillard, «Convergence, transferts et traduction : les apports de la comparaison transnationale », Gouvernement et action publique, 2 (3), 2013, p. 377-393 ; A. Vauchez, « Le prisme circulatoire... », art. cité.

${ }^{31}$ Antoaneta L. Dimitrova, Bernard Steunenberg, « The Power of Implementers : A Three-Level Game Model of Compliance With EU Policy and Its Application To Cultural Heritage », Journal of European Public Policy, 2017, 24 (8), p. 1211-1232.

${ }^{32}$ D. Stone, « Private Philanthropy or Policy Transfer... », art. cité.

33 David P. Dolowitz, Rodica Plugaru, Sabine Saurugger, «The Process of Transfer: The Micro- 
Une partie de la sociologie de la circulation des normes a accordé une attention soutenue aux passeurs, mais cette attention souffre de plusieurs biais. Le premier est un biais élitiste. Consacrée à la circulation de biens élaborés, pris dans des circuits particulièrement qualifiés, cette sociologie des passeurs ou des « courtiers de l'international », pour reprendre l'expression fameuse d'Y. Dezalay ${ }^{34}$, concentre le regard sur les positions sommitales des appareils d'État et des organisations internationales (fondations au premier rang) ou des organismes universitaires dont ils sont, pour cette sociologie, les relais essentiels dès lors que, comme le soulignait Pierre Bourdieu, «la référence à l'universel, au juste, est l'arme par excellence $^{35} »$. La circulation des normes et des politiques s'effectue en effet par le biais d'acteurs dont il est raisonnable de penser qu'ils disposent des ressources nécessaires à la circulation de ces biens immatériels complexes; aussi n'est-on pas surpris de retrouver juristes de droit international, chargés de stratégies internationales ou de développement international au sein de sociétés privées, dont les ressources sont telles qu'elles pèsent sur la configuration et les règles des marchés dans lesquels elles opèrent, comme les géants de l'Internet ou du médicament, et plus généralement acteurs multipositionnés et bien nés ${ }^{36}$, «héritiers cosmopolites » comme le suggère Y. Dezalay ${ }^{37}$, passeurs de normes et de règles qui ont pour vertu incidente de convertir leurs positions en rentes de situation.

La sociologie des mobilisations transnationales indique également que ces jeux Influences of Power, Time and Learning », Public Policy and Administration, 35 (4), 2020, p. 445-464.

34 Yves Dezalay, «Les courtiers de l'international: héritiers cosmopolites, mercenaires de l'impérialisme et missionnaires de l'universel », Actes de la recherche en sciences sociales, 151-152, 2004, p. 435. Il est surprenant, à l'examen, de constater que le terme « courtier de l'international » ne donne pas lieu à une défense de l'importation du terme de « courtier » depuis le champ de l'activité boursière ou commerciale vers le champ de la sociologie : le terme n'est d'ailleurs employé qu'une seule fois dans le corps du texte.

${ }^{35}$ Pierre Bourdieu, Raisons pratiques. Sur la théorie de l'action, Paris, Seuil, 1994, ici p. 242.

36 Thibaud Boncourt, «Acteurs multipositionnés et fabrique du transnational : la création du European Consortium for Political Research », Critique internationale, 59, 2013, p. 17-32 ; Elisabeth Anderson, «Policy Entrepreneurs and The Origins of The Regulatory Welfare State : Child Labor Reform in Nineteenth-Century Europe », American Sociological Review, 83 (1), 2018, p. 173-211. Un cas limite de ces activités d'intermédiation est celui des grandes organisations philanthropiques qui, à l'image des fondations Soros ou Gates, financent des bureaucraties non-gouvernementales aptes à imposer des agendas politiques nationaux ou internationaux, voire (beaucoup plus rarement néanmoins) des clivages ou des forces politiques à l'échelle nationale (Peter Haegel, «The Power of Billionaire Philanthropy in World Politics», dans Anne Monier, Sylvain Lefèvre (dir.), Philanthropie et démocratie, Paris, PUF, à paraître.

${ }^{37}$ Y. Dezalay, « Les courtiers de l'international... », art. cité. 
transnationaux de confection et de circulation de normes ou de politiques publiques s'effectuent aussi à des échelons intermédiaires, voire inférieurs. Johanna Siméant et Pascal Dauvin ont montré ce que l'engagement dans l'humanitaire, voire la genèse de ce domaine d'action, devait à des familles juives dont l'expérience diasporique avait permis la constitution de dispositions à l'action humanitaire internationale, sans pour autant que celle-ci soit le vecteur de stratégies de conquête ou de défense de positions dominantes acquises dans l'espace national ${ }^{38}$. Les travailleurs humanitaires restent des agents sociaux qui alternent «terrain » et «siège », entrent en transaction avec des interlocuteurs étrangers détenteurs de pouvoirs nationaux, locaux, voire seulement conjoncturels (le potentat d'une localité par lequel passent tous les arrangements nécessaires à l'organisation d'un camp de réfugiés ou d'une distribution de soins). Ils entrent également dans le jeu de l'élaboration de la norme nationale en matière, par exemple, d'aide au développement ou de brevets sur le médicament dans leur propre espace national, sans nécessairement y occuper des positions éminentes, ni se livrer à quelque « double jeu ${ }^{39}$ » propre aux courtiers de l'international. Les passeurs que sont les salariés de l'humanitaire contribuent, avec (ou contre) d'autres, dans un champ concurrentiel, à la construction des normes de l'intervention internationale et du développement, tout en occupant des positions secondaires dans leurs propres organisations nationales et en tentant d'y valoriser leurs convictions propres.

Le biais élitiste ne consiste pas seulement à supposer que les grandes choses de ce monde sont nécessairement le fait des grands (hommes), mais aussi à supposer que les positions internationales sont, du fait même qu'elles sont internationales, plus enviables et enviées, plus convoitées et plus puissantes. Le recul historique permet aujourd'hui de mieux juger, par exemple, de ces officiers de liaison tenus pour élites transnationales de leurs administrations d'origine, aptes à construire des «polices en réseaux ${ }^{40}$ » en vue d'une Europe policière échappant aux États. S'il est évident que dans tout un ensemble de domaines ces acteurs ont permis l'échange d'informations, mais surtout, en réalité, l'échange d'idées et de conceptions, la portée réelle de leur implication est restée très limitée : les polices ne mettent en réseau qu'une infime partie de leurs savoirs et de leurs fichiers, et leurs conditions

\footnotetext{
${ }^{38}$ Pascal Dauvin, Johanna Siméant, Le travail humanitaire. Les acteurs des ONG, du siège au terrain,
} Paris, Presses de Sciences Po, 2002. Voir aussi les rooted cosmopolitans de Donatella della Porta, Sidney Tarrow (dir.), Transnational Protest and Global Activism, Lanham, Rowman \& Littlefield Publishers, 2005.

${ }^{39}$ Y. Dezalay, « Les courtiers de l'international... », art. cité.

${ }^{40}$ Didier Bigo, Polices en réseaux. L'expérience européenne, Paris, Presses de Sciences Po, 1996. 
d'exercice sont toujours très largement déterminées par les États nationaux ${ }^{41}$. À l'inverse, des normes de politique publique promises à une intense circulation, comme la notion de «tolérance zéro » en matière de lutte contre la délinquance, ont plutôt émergé de la compétition politique interne, portée par des acteurs de droite défendant dans (contre) leur camp des options atlantistes et des acteurs de gauche défendant dans (contre) leur camp des positions dures de politique criminelle, d'ailleurs assez éloignées du modèle new-yorkais ${ }^{42}$. Nulle élite en jeu ici, mais des acteurs politiques qui, à tous les niveaux de leurs organisations respectives, emploient diverses contingences pour se saisir d'une notion en circulation, s'en faire les passeurs et en tirer des profits de distinction dans leur champ propre. À tous les niveaux de leurs organisations, nous y insistons, car une vision trop élito-centrée tend à négliger les échelles intermédiaires telles que les collectivités territoriales dans la circulation des normes, manifeste par exemple dans le domaine de la santé publique lors de la diffusion en Europe des politiques de réduction des risques ${ }^{43}$ et de manière générale dans la diffusion des politiques de prévention, où les acteurs intermédiaires du champ, voire extérieurs au champ de la santé publique, ont contribué à la diffusion des normes de santé publique ${ }^{44}$. C'est donc à une attention plus particulièrement portée sur les agents de diffusion et de circulation que nous appelons ici, afin principalement d'éviter le biais de séduction exercé par la sociologie des élites, à qui l'on prête trop vite gain, succès et réalisation des ambitions.

Analyser les passeurs pour dépasser le biais institutionnaliste

Dans la littérature disponible, les acteurs intermédiaires se caractérisent par leurs capacités à s'affranchir momentanément des dépendances horizontales et verticales que leur

${ }^{41}$ Fabien Jobard, Jacques de Maillard, Sociologie de la police. Politiques, organisations, réformes, Paris, Armand Colin, 2015, voir p. 234-236.

42 Jacques de Maillard, Tanguy Le Goff, «La tolérance zéro en France : succès d'un slogan, illusion d'un transfert », Revue française de science politique, 59 (4), août 2009, p. 655-679.

43 Olivier Fillieule, Fabien Jobard, «Action publique sous dépendance : conditions et effets du changement de paradigme dans la lutte contre la délinquance associée à la drogue en Europe », Revue française de science politique, 49 (6), décembre 1999, p. 803-834.

${ }^{44}$ Luc Berlivet, «Une biopolitique de l'éducation pour la santé », dans Didier Fassin, Dominique Memmi (dir.), Le gouvernement des corps, Paris, Éditions de l'EHESS, 2004, p. 37-75 ; Gabriel Girard, Les homosexuels et le risque du sida. Individu, communauté et prévention, Rennes, Presses universitaires de Rennes, 2013. 
statut leur impose ${ }^{45}$. Ils développent des savoirs portant sur l'utilisation des règles propres aux environnements institutionnels auxquels ils appartiennent, et mobilisent et contrôlent des images légitimes des groupes et des univers qu'ils mettent en contact. La force d'une telle approche est de mettre en avant le rôle central d'acteurs dont la principale caractéristique est leur multipositionnement - apport ancien de la sociologie des organisations ${ }^{46}$ - dans la circulation des savoirs et des normes entre institutions et entre niveaux de gouvernance ${ }^{47}$. En somme, ces analyses ont permis d'identifier le jeu d'acteurs spécifiques comme rouages indispensables dans une constellation institutionnelle complexe.

La limite de cette approche réside néanmoins dans son biais institutionnaliste. Les acteurs intermédiaires sont souvent vus comme des médiateurs ou comme des relais entre groupes dont les intérêts divergent. La sociologie du rôle de ces acteurs est indexée à une perception des sociétés modernes caractérisée par une forte sectorisation des activités, qui nécessite des liens entre les sous-secteurs. Les acteurs intermédiaires sont des «acteurs relais $^{48} »$, «brokers ${ }^{49} »$ ou «médiateurs ${ }^{50} »$, dont l'activité consiste à faire émerger un référentiel apte à donner un sens à l'ensemble des sous-secteurs et donc à la société tout entière $^{51}$. Selon cette logique, le médiateur est un acteur clé dans l'élaboration d'un référentiel global justifiant une attention soutenue à son rôle dans l'émergence d'un cadre cognitif commun. Les travaux consacrés aux policy brokers ${ }^{52}$ permettent ainsi d'expliquer la

${ }^{45}$ O. Nay, A. Smith (dir.), Le gouvernement du compromis..., op. cit.

${ }^{46}$ Michel Crozier, Erhard Friedberg, L'acteur et le système. Les contraintes de l'action collective, Paris, Seuil, 1977.

${ }^{47}$ Frederic O’Neill, « Health : The "Internal Market” and The Reform of The National Health Service », dans David Dolowitz(dir.), Policy Transfer and British Social Policy. Learning from The USA ?, Buckingham/Philadelphie, Open University Press, 2000, p. 59-76; Maurice Rouzeau, «Les experts et la politique de la ville : circulation des idées, mobilisation des connaissances et animation transactionnelle », dans Steve Jacob, Jean-Louis Genard (dir.), Expertise et action publique, Bruxelles, Éditions de l'Université de Bruxelles, 2004, p. 65-80.

${ }^{48}$ M. Crozier, E. Friedberg, L'acteur et le système..., op. cit.

${ }^{49}$ Karin Ingold, Frédéric Varone, «Treating Policy Brokers Seriously : Evidence from The Climate Policy », Journal of Public Administration Research and Theory, 22 (2), 2011, p. 319-346.

${ }^{50}$ Bruno Jobert, Pierre Muller, L'État en action. Politiques publiques et corporatisme, Paris, PUF, 1987.

${ }^{51}$ Ibid. ; Pierre Muller, «Les politiques publiques comme construction d'un rapport au monde », dans Alain Faure, Gilles Pollet, Philippe Warin (dir.), La construction du sens dans les politiques publiques. Débats autour de la notion de référentiel, Paris, L’Harmattan, 1995, p. 153-179.

${ }^{52}$ K. Ingold, F. Varone, « Treating Policy Brokers Seriously... », art. cité, exposent bien les limites de la 
création d'une coalition d'acteurs autour de nouvelles idées, qui rend possible le changement d'orientation d'une politique publique donnée. L'enjeu principal de l'analyse reste de comprendre les jeux institutionnels et, de fait, la circulation de savoirs ou de normes n'est un objet d'intérêt scientifique que lorsqu'elle traverse des espaces institutionnels distincts, qu'elle contribue à briser l'étanchéité entre les secteurs sociaux, les institutions, les espaces professionnels (ou échoue à le faire). Et l'analyse repose toujours sur l'hypothèse selon laquelle une médiation doit avoir lieu pour concilier des mondes différents, réduisant l'acteur à son rôle institutionnel. La position de Paul Williams, dans son texte «Sommes-nous tous des passe-murailles ? ( «We Are All Boundary Spanners Now ? »), offre un bon exemple de l'institutionnalisme qui marque la grille de perception sociologique des acteurs. Son questionnement est en effet le suivant :

«Du fait de la complexité et de l'interrelation des questions de politique publique que doivent traiter les services publics, une proportion croissante du travail de nombreux praticiens, managers ou directeurs, est consacrée au passage des frontières professionnelles, devenu partie intégrale de leurs postes respectifs [...]. La discussion consiste alors à déterminer combien ces rôles et compétences de passe-murailles sont similaires, ou différents, à ceux employés autrefois dans la conduite des organismes publics traditionnels [...] et à savoir dans quelle mesure on pourrait définir désormais la plupart des agents publics comme des passe-murailles ${ }^{53}$. »

À l'encontre de ces travaux concevant les opérateurs de transfert seulement comme des représentants de leur institution, la notion de passeur permet de sociologiser et désinstitutionnaliser l'analyse des transferts.

\section{Les apports d'une analyse par les passeurs}

notion de policy broker, en soulignant combien ces acteurs sont essentiellement guidés par des logiques de maximisation des rétributions tirés de leur position d’intermédiaire. Si Karine Ingold et Frédéric Varone reconnaissent une diversité des statuts et des capacités possibles, ils estiment que la littérature reconnaît toujours à ces policy brokers une forte capacité à exploiter les logiques d'opportunité et/ou les faiblesses structurelles des institutions publiques, en s'appuyant sur une forte dotation en capitaux personnels, au premier rang desquels leur capacité à diriger, voire manipuler, des personnels. On retrouve ces dimensions dans l'ouvrage de Jeremy Boissevain que Thomas Bierschenk, Jean-Pierre Chauveau et Jean-Pierre Olivier de Sardan identifient comme l'un des premiers proposant, en anthropologie politique, l'usage du terme de courtier.

${ }^{53}$ Paul Williams, «We Are All Boundary Spanners Now ? », International Journal of Public Sector Management, 26 (1), 2013, p. 19 (traduction par les auteurs). Sur la notion de «boundary spanners », voir José Nederhand, Martijn Van Der Steen, Mark Van Twist, «Boundary-Spanning Strategies For Aligning Institutional Logics : A Typology », Local Government Studies, 45 (2), 2019, p. 219-240. 
Les différentes contributions sont consacrées aux acteurs qui ont, au préalable, été identifiés comme passeurs. L'avantage à étudier leurs différentes propriétés est de les articuler aux contextes institutionnels dans lesquels ils évoluent. Pour ce faire, nous avons choisi de présenter des travaux assurant une diversité aussi bien des échelles d'analyse que des zones géographiques étudiées. En nous intéressant aux croyances et convictions de ces acteurs, il sera possible de saisir les conditions de possibilité de la circulation (même provisoire et fragile). Ainsi, nous n'entrevoyons pas les passeurs de savoirs ou de normes comme incarnant une fonction, mais cherchons à identifier les investissements de rôles de la part d'acteurs de natures très diverses.

Notre ambition est donc, par le biais de contributions venant des recherches en sociologie de l'international et en sociologie de l'action publique, de proposer une première déclinaison des catégories de passeurs, en stylisant une matrice d'oppositions entre les différentes figures sociologiques qu'ils peuvent incarner. Nous serons alors à même de montrer quelques avantages comparatifs de la notion de passeur.

Identification d'une matrice d'oppositions

Forts du constat que les passeurs ne représentent pas une catégorie d'acteurs homogènes et que leurs motivations et trajectoires peuvent avoir des implications significatives sur la manière dont les savoirs, les normes circulent, un effort de classement des passeurs est nécessaire ${ }^{54}$. Notre dossier permet d'identifier trois principales oppositions entre passeurs.

La première procède d'une analyse des façons dont les acteurs deviennent passeurs. Certains le deviennent car leur activité professionnelle l'exige alors que d'autres s'engagent dans des activités de passage de leur propre volonté. Une première opposition se fait ainsi jour entre «passeurs de profession » et « passeurs de conviction ». Elle ne signifie pas que les premiers ne sont pas convaincus du bien-fondé de leur activité ou que les seconds n'agiraient que par idéologie : l'état d'esprit des acteurs n'est pas ici le critère discriminant, mais la différence entre rôle et statut. Les passeurs de conviction doivent «prendre un rôle », tandis que le rôle qu'exercent les passeurs de profession est inscrit dans le statut que leur octroie leur institution d'appartenance - dans leur cas, le rôle n'est pas à prendre, même si les modalités d'interprétation sont bien sûr, et dans les limitent que fixent leurs statuts, à la

\footnotetext{
${ }^{54}$ Voir aussi, dans une même démarche, J. Lagroye, « Être du métier », art. cité, p. 9.
} 
discrétion des passeurs. . Pour les passeurs de profession, les activités de transfert font partie intégrante et officielle de leur travail rémunéré. Les passeurs présentés dans la contribution d’Ulrike Zeigermann et la majorité de ceux décrits par Irina Mützelburg et Jérémy Geeraert se caractérisent par un rôle de passeur qui est essentiellement le produit de la position qu'ils occupent dans leur institution, position qui de surcroît marque la manière dont ils font circuler le savoir et définit en grande partie leurs marges de manœuvre. En revanche, pour les passeurs de conviction, les activités de transfert se font en plus de ce que leur demande leur travail formel. Leur engagement pour les transferts peut avoir lieu pendant et hors du temps de travail. Une partie des passeurs étudiés par I. Mützelburg endossent ainsi un rôle de passeur en plus de leurs activités d'agents administratifs; leurs supérieurs ne le leur demandent pas et parfois n'apprécient pas leur engagement. L'article de Bénédicte Laumond introduit, quant à lui, la figure d'un magistrat qui émerge comme l'un des pionniers de la réorientation de la politique publique luttant contre l'extrémisme de droite dans le Land du Brandebourg en Allemagne. Le statut de magistrat et les rétributions possibles mais très incertaines d'une réforme de la politique de lutte contre l'extrémisme de droite ne suffisent pas à comprendre pourquoi cet acteur devient à partir du milieu des années 1990 un promoteur et un passeur de savoirs ; son engagement repose également sur des convictions personnelles. C'est également le cas du médecin ayant importé à l'hôpital public le modèle des consultations pluriprofessionnelles dédiées à la prise en charge de la précarité dans l'article de Jérémy Geeraert. Sa position de chef de service dans son hôpital, son double ancrage dans le monde hospitalier et le monde associatif et ses relations dans le monde politique ont facilité le travail de circulation de la norme entrepris par ce passeur, sans non plus bien sûr que la création de cette politique publique soit réductible au multipositionnement d'un seul acteur. Essentielles à la compréhension de sa prise de rôle de passeur sont ses convictions personnelles, marquées par une socialisation politique dans les milieux de la médecine militante de gauche dans les années 1970.

Les deux autres oppositions identifiées dans ce dossier sont d'ordre institutionnel ${ }^{55}$. Elles permettent de préciser ce qu'être passeur signifie sur le terrain. D'une part, nous

55 Voir aussi les recherches sur les ressources institutionnelles et les stratégies des «policy entrepreneurs »: Elin Lerum Boasson, Dave Huitema, «Climate Governance Entrepreneurship : Emerging Findings and a New Research Agenda », Environment and Planning C: Politics and Space, 35 (8), 2017, p. 1343-1361 ; Neil Fligstein, Doug McAdam, A Theory of Fields, Oxford, Oxford University Press, 2012 ; D. Stone, « Transnational Policy... », art. cité ; Id., « Private Philanthropy or Policy Transfer ? », art. cité. 
identifions des passeurs de l'intérieur et des passeurs de l'extérieur. Alors que les premiers - comme les médecins dans la contribution de J. Geeraert - sont déjà installés dans leur institution, les seconds interviennent dans l'institution en raison de la nécessité d'y introduire un savoir venu d'ailleurs. Cette opposition déduite des trajectoires professionnelles permet d'identifier des logiques variées de passage des normes au sein d'une même institution, indexées chacune à l'identité même des passeurs. En termes théoriques, elle permet d'identifier différents modes d'investissement du rôle de passeur. On constate ainsi parfois la capacité des passeurs eux-mêmes à jouer avec ces oppositions venant structurer leur univers professionnel : I. Mützelburg explique comment des passeurs professionnels, au départ extérieurs à l'institution, parviennent à intégrer l'administration nationale - la cible de leur entreprise de transfert. À force de côtoyer le personnel administratif dans cette institution étatique, ils réussissent à brouiller les lignes entre intérieur et extérieur, ce qui fait partie intégrante de leur stratégie de transfert. À l'inverse, B. Laumond décrit l'isolement des passeurs du Brandebourg dans leur environnement institutionnel, les menant à sortir de leurs institutions respectives en œuvrant à la formation d'un réseau de passeurs. Par contraste, les acteurs du secrétariat général de l'OCDE étudiés par U. Zeigermann sont d'abord des passeurs de l'intérieur puisqu'ils travaillent à la définition d'une norme qui est diffusée depuis leur institution. Le réseau transdisciplinaire et transnational qu'ils construisent et dont ils font partie est, quant à lui, constitué de passeurs de l'extérieur qui vont diffuser la norme dans d'autres institutions. Les passages d'une même norme s'effectuent ainsi à plusieurs niveaux, à la fois vers l'intérieur et vers l'extérieur selon les statuts des acteurs considérés et selon le moment dans le processus de passage.

Les textes que nous réunissons mettent ainsi en lumière le fait que les passeurs euxmêmes butent sur les lignes de démarcation qui séparent le monde vers lequel ils entendent agir et le monde d'où ils agissent : le statut d'externes aux institutions visées les éloigne bien sûr des ressources nécessaires, du fait de la méfiance suscitée par leur intervention, jusqu'à la peur de l'ingérence. Ils tentent alors une double position, qui mêle aux ressources externes (par exemple des moyens financiers mais aussi l'indépendance des contraintes et voies hiérarchiques internes) les gains tirés de la familiarité et de la proximité avec les acteurs visés.

Enfin, nous distinguons au sein de leurs institutions respectives les passeurs monopositionnés et les passeurs multipositionnés. Si l'observation de ces derniers est peu 
étonnante et vient faire écho à la littérature sur les transferts soulignant la capacité de ces acteurs intermédiaires à faire passer le savoir ou la norme d'un espace à l'autre ${ }^{56}$, l'identification de passeurs monopositionnés est plus inattendue. Les passeurs du secrétariat général de l'OCDE, chez U. Zeigermann, ne sont pas multipositionnés au départ: ils diffusent la notion de «cohérence » entre différents départements thématiques et unités dans la hiérarchie de l'organisation. Dans le contexte de son analyse menée à l'échelle de l'institution hospitalière, J. Geereart montre que les employés des permanences d'accès aux soins de santé (PASS) dans la seconde phase du transfert sont également monopositionnés. C'est parce que la norme importée n'est pas encore stabilisée que ces employés hospitaliers endossent des rôles de passeur à l'intérieur de leur institution. Cette dernière opposition nous permet d'un point de vue théorique de caractériser, en fonction du terrain étudié, la nécessité (ou l'absence de nécessité) pour les acteurs de se déplacer avec les normes qu'ils diffusent, afin justement de faire circuler le savoir ou la norme qu'ils promeuvent. Quoi qu'il en soit, l'une des conclusions que les textes que nous présentons ici permettent de formuler est l'observation selon laquelle les passeurs ne sont ni nécessairement dominants au sein de leur institution, ni nécessairement sécants pour faire circuler des normes. L'essentiel tiendrait à ce que, à leur échelle, endosser ce rôle est opportun (et la notion de fenêtre d'opportunité est ici bien sûr centrale), voire vital (lorsque, sans appropriation et diffusion de normes ou d'idées nouvelles c'est la survie des statuts individuels ou de l'organisation qui est en jeu).

Ces trois paires d'opposition permettent d'ordonner nos passeurs en une matrice facilitant l'analyse. On constate en effet qu'il est possible de combiner différemment les propriétés précédemment énoncées : on peut ainsi être un passeur de conviction, de l'intérieur et monopositionné comme le sont une partie des médecins des PASS chez J. Geeraert. On observe aussi des passeurs de conviction, multipositionnés, d'abord de l'intérieur puis allant vers l'extérieur comme le procureur général ou la déléguée en charge des questions migratoires dans l'article de B. Laumond. Ces propriétés peuvent changer au cours du temps ou selon les situations, comme l'illustre l'article d'U. Zeigermann. La force de cette matrice oppositionnelle est de pouvoir mener à des comparaisons sur un même terrain : ainsi I. Mützelburg présente-t-elle des passeurs dont les positionnements institutionnels sont variés. Une telle matrice oppositionnelle rend possible la comparaison entre terrains et donc entre la

${ }^{56}$ Sur la notion de «passe-murailles » (boundary spanners) actuellement à l'usage dans une partie de la littérature, voir O. Nay, A. Smith (dir.), Le gouvernement du compromis..., op. cit.; et l'article d'Ulrike Zeigermann dans ce dossier. 
diffusion de différents savoirs ayant lieu entre différentes institutions. Elle permet également, comme c'est le cas chez J. Geeraert, de distinguer des modes de diffusion d'une norme au sein d'une même institution selon les temporalités du transfert et les positionnements institutionnels des passeurs. Au contraire, la contribution de B. Laumond donne à voir la multiplication de passeurs mus par des convictions communes dans différentes sphères institutionnelles et dans le temps qui, tous, œuvrent à la réorientation d'une politique publique. Selon leur statut professionnel, leurs convictions et leur position institutionnelle, les passeurs de normes, d'idées et d'action publique de ce dossier offrent une palette de rôles sociaux.

De quelques avantages comparatifs du concept de passeur

\section{Contre l'assignation statutaire : la complexité des rôles}

La notion de passeur permet de nous éloigner d'une littérature dans laquelle les transferts relèvent d'une dynamique top-down et linéaire. Au contraire, les textes que nous présentons insistent sur la complexité des processus de circulation, dans lesquels différents types d'acteurs sont impliqués. Ce faisant, nous introduisons une nuance par rapport à la sociologie des acteurs des transferts conçue en termes de policy entrepreneurs. Pour cette littérature, l'organisation dont relève l'acteur ou plus largement l'environnement institutionnel de ce dernier est primordiale ${ }^{57}$. En réalité, une approche plus microsociologique qui fait la part belle à l'environnement immédiat des acteurs concernés, les passeurs, montre qu'au sein d'une même organisation ou d'un même environnement institutionnel des rôles différents peuvent être investis, porteurs d'influences différentes sur les transferts et circulations alors observés - raison pour laquelle nous ne parlons pas de policy entrepreneurs, mais de simples passeurs ${ }^{58}$.

\section{Faire leur place aux acteurs secondaires}

Ce dossier vient par ailleurs invalider la simple opposition entre des transferts de normes qui seraient volontaires et d'autres qui seraient au contraire imposés aux acteurs intermédiaires. Une sociologie des passeurs permet en effet de montrer que ces derniers

${ }^{57}$ Marcel Braun, «The Evolution of Emissions Trading in The European Union : The Role of Policy Networks, Knowledge and Policy Entrepreneurs », Accounting, Organizations and Society, 34 (3-4), 2009, p. $469-487$.

${ }^{58}$ Dans le même esprit, voir D. Stone, « Transnational Policy... », art. cité. 
peuvent, malgré la contrainte institutionnelle ${ }^{59}$, s'engager volontairement et de manière différenciée dans la circulation de savoirs. On songe alors aux acteurs qui tentent de survivre au sein de l'institution hospitalière chez $\mathrm{J}$. Geeraert ou à ceux qui sont des professionnels du passage et se placent au-delà de la simple opposition entre volontarisme et contrainte, comme chez U. Zeigermann et I. Mützelburg. On s'écarte donc d'analyses influencées par les courants néo-institutionnalistes pensant le rôle des acteurs passeurs comme celui de représentants de leur institution (y occupant une place centrale ou étant au contraire des marginaux-sécants) puisque les dynamiques de circulation des savoirs sont multiples, y compris au sein d'un même environnement institutionnel.

Ce dossier s'attache aussi à montrer que la circulation de savoirs, dans une institution, relève de mécanismes variés et que les passeurs, leurs trajectoires, leurs choix doivent être comparés les uns aux autres afin de mieux rendre compte des conditions de possibilité du passage de normes et de savoirs. En centrant l'analyse sur les acteurs (sans présumer de leur positionnement institutionnel pour atteindre la position de passeur), on se donne les moyens de tenir compte à la fois des intérêts, des rôles et/ou des effets des institutions, sans avoir à choisir un angle d'approche particulier, tout en structurant l'analyse au plus près des réalités observées sur le terrain. Dans la même veine, l'étude des passeurs permet d'intégrer à l'analyse tant les courtiers de l'international que les acteurs intermédiaires et ainsi d'aborder les circulations de savoirs à différentes échelles et de les comparer entre elles. Il est alors envisageable de comparer les conditions de passage aux niveaux européen (I. Mützelburg) ou transnational (U. Zeigermann) à celles aux niveaux régional (B. Laumond) ou local (J. Geeraert).

\section{Le rôle central de la temporalité}

Analyser la circulation au prisme des passeurs met en lumière le rôle central de la temporalité, principalement de la durée, dans l'identification des interactions d'acteurs décisives pour que le transfert de savoirs puisse être opéré, ce dans la lignée de travaux récents ${ }^{60}$. Notre dossier montre en effet qu'intégrer la dimension temporelle dans le

${ }^{59}$ Ibid. ; M. Braun, « The Evolution of Emissions... », art. cité.

${ }^{60}$ Tels que D. Dolowitz, R. Plugaru, S. Saurugger. «The Process of Transfer... », art. cité ; Philipp Pattberg, «The Emergence of Carbon Disclosure... », art. cité, sur la temporalité des processus de transfert; C. Walker, C. Mavrot, D. Dolowitz, «From state to market: regulation of road transport in Australia and South Africa », dans M. Hadjiisky, L. Alexander Pal, C. Walker, Public Policy Transfer..., 
développement d'une sociologie des passeurs rend possible une meilleure appréhension de l'articulation entre les stratégies déployées ou envisagées et les types de passeurs. Elle permet également de soutenir que le passage de normes ne peut avoir lieu par inadvertance.

Les acteurs œuvrant à la diffusion des normes internationales et européennes de l'asile en Ukraine chez I. Mützelburg organisent le transfert de normes de manière stratégique ${ }^{61}$. Certaines organisations investissent dans des programmes de formation d'intervenants qui assumeront le rôle de passeurs dans l'univers cible des transferts ${ }^{62}$. La stratégie ainsi mise en œuvre s'appuie sur des ressources suffisantes pour jouer sur la durée, en distinguant un temps d'apprentissage du rôle de passeur et un temps de mise en œuvre du rôle appris. Dans d'autres contextes, la durée s'impose aux acteurs. Ainsi, les structures du soin de la précarité étudiées par J. Geeraert mettent en lumière le rôle de deux types de passeurs selon le moment du transfert. Dans un premier temps, des acteurs bénéficiant de capitaux suffisants peuvent par leurs seules ressources assurer l'exportation des normes qu'ils promeuvent vers l'institution hospitalière. Une fois ces acteurs retirés du jeu, ces institutions ne bénéficient plus de leurs ressources et d'autres passeurs entrent en jeu, dans une phase d'institutionnalisation, qui compensent la précarité de leurs capitaux propres en construisant des alliances, des forums, des groupes d'intérêt. Dans un sens voisin, B. Laumond démontre que la durée sur laquelle se déploie l'action des passeurs du Brandebourg met en scène deux générations distinctes d'acteurs, les premiers disposant de ressources fortes et autonomes, les seconds étant engagés dans une dynamique de professionnalisation, toutes deux étant au final indispensables au passage d'un savoir innovant dans une politique publique ancienne. De même, U. Zeigermann révèle que les transferts sont largement déterminés par la capacité des passeurs à user des moments comme autant de fenêtres d'opportunité afin de promouvoir leur savoir. Enfin, chez I. Mützelburg, le temps intervient également comme risque d'échec des stratégies de transfert: alors que ses passeurs déploient des tactiques de rapprochements individuel, amical, familial, avec les acteurs de l'administration ukrainienne qu'ils souhaitent enrôler, ceux-ci sont dépendants de rythmes institutionnels (turn-over des personnels) qui sont toujours susceptibles de briser les efforts engagés sur la durée par les ONG externes ou, si les liens personnels ne sont pas rompus, de les priver de toute valeur d'usage ${ }^{63}$.

op. cit., p. 79-100, travaillant sur les processus de feedback et d'apprentissage.

\footnotetext{
${ }^{61}$ S. Jacquot, C. Woll (dir.), Les usages de l'Europe..., op. cit.

${ }^{62}$ P. Williams, «We Are All Boundary Spanners Now ? », art. cité.

${ }^{63}$ Sur le lien entre le timing et les conditions de réalisation de l'action, voir Donald Miller, « Political
} 


\section{Des passeurs à la configuration?}

Enfin, l'attention portée aux passeurs pourrait permettre, dans des études ultérieures, d'approfondir les facteurs qui font la fragilité ou la robustesse des transferts de normes et des circulations de savoirs. Les textes de ce dossier suggèrent que la constellation des acteurs, leurs intérêts, idées, stratégies et ressources, pourraient expliquer les gradients de fragilité (ou de solidité) observables dans la circulation des idées, des normes ou des politiques publiques. Ainsi, la stabilité des statuts institutionnels des passeurs et le fait que ceux-ci soient durablement ancrés dans leur arène institutionnelle pourraient contribuer à expliquer la stabilité des transferts (contribution d'U. Zeigermann et de B. Laumond). En revanche, le fait que des passeurs professionnels ne pratiquent le passage de savoirs qu'en raison d'une position rémunérée implique que dans des situations marquées par l'instabilité des financements, la circulation est fragile (contribution d'I. Mützelburg). Une manière d'approfondir notre étude serait de tester l'hypothèse d'un éventuel lien entre la configuration que dessinent les interventions des passeurs et celle de la robustesse des transferts de savoirs ou de normes, de s'attacher aux notions d'apprentissage et de feedback qui sont au cœur des récentes contributions sur ce domaine d'étude des politiques publiques.

\section{Les contributions du dossier}

Ce dossier procède des réflexions et échanges du groupe de travail Action publique et circulations des savoirs du Centre Marc Bloch à Berlin, animé par Fabien Jobard et U. Zeigermann de 2015 à 2018. Dans sa contribution, U. Zeigermann étudie la diffusion de la norme «cohérence des politiques pour un développement durable». Elle restitue la circulation de cette norme non pas telle que portée par la division dédiée au secrétariat général de l'OCDE, mais par les acteurs qui s'en sont investis : les passeurs transnationaux. Au-delà de leurs statuts partagés à l'OCDE, ceux-ci forment un réseau international et transdisciplinaire d'acteurs agissant autour des questions de cohérence. L'article souligne les interactions dynamiques entre ces passeurs et l'institution dont ils relèvent. U. Zeigermann montre notamment comment les acteurs du secrétariat de l'OCDE qui œuvrent à la promotion d'une norme de politique publique sont dotés d'un capital réputationnel fort, appuyé sur la

Time: The Problem of Timing and Chance », Time and Society, 2 (2), 1993, p. 179-197 ; Fabien Jobard, «Usages et ruses des temps : l'unification des polices berlinoises après 1989 », Revue française de science politique, 53 (3), juin 2003, p. 351-381. 
légitimité du savoir dont ils sont censés disposer. Mais aussi comment, dans un espace de concurrence autour de la définition même de la norme à diffuser, ils évoluent d'une position charnière $d^{\prime}$ " intermédiaire de profession » vers celle de «médiateur multipositionné ». Ainsi, ce sont bien les interactions à l'intérieur d'un environnement politique qui, à la fois, influent sur l'autonomie des passeurs dans l'administration publique et transforment leurs fonctions dans les processus de circulation des savoirs politiques (celles de mettre en relation des entités, de rendre audibles des discours ou des pratiques, mais également celle de traduire le savoir transféré et donc potentiellement de le transformer).

I. Mützelburg, quant à elle, analyse les entreprises d'acteurs internationaux visant à diffuser des normes d'asile en Ukraine. Elle met ainsi au jour une «chaîne de transferts » impliquant une diversité d'acteurs, liés par des relations de dépendance, voire de délégation, les uns passeurs de profession, les autres devenus passeurs de conviction par les interactions avec les premiers. Elle montre ainsi comment le bureau du Haut-Commissariat des Nations unies pour les réfugiés (HCR) à Kiev produit des entrepreneurs de normes locaux, des ONG dont le personnel est rémunéré pour transférer des standards internationaux en matière d'asile vers des agents étatiques. Ces passeurs professionnels n'avaient pas d'engagement militant préalable dans ce secteur et leurs activités de transfert dépendent de la disponibilité de financements internationaux. Cherchant à faire passer les normes internationales aux agents étatiques ukrainiens, les employés d'ONG et d'organisations internationales en Ukraine cherchent à brouiller les frontières entre extérieur et intérieur de la fonction publique en établissant des liens informels, voire amicaux, avec les fonctionnaires, par exemple en plaçant certains de leurs employés quelques jours par semaine au sein de l'administration ukrainienne, afin de les familiariser aux attentes et contraintes des destinataires et, inversement, de faire de certains agents étatiques des passeurs de normes au sein même de leur institution, leur délégant ainsi l'activité de transfert.

B. Laumond s'intéresse, pour sa part, à des passeurs qui promeuvent une politique préventive permettant de lutter contre l'extrémisme de droite dans l'État du Brandebourg en Allemagne à partir des années 1990. Les savoirs qui sont transférés ne sont pas importés d'un espace géographique extérieur à l'Allemagne et l'enjeu est de déterminer le rôle des passeurs dans une dynamique circulatoire intervenant au sein d'une polity. Elle montre que ces acteurs de la première génération, jouissant d'un statut à la fois protégé et enviable, deviennent passeurs par des convictions fortes que la sociologie de leurs intérêts purs ne permet pas de comprendre. Leur objectif est l'introduction de mesures préventives dans la politique publique contre l'extrémisme de droite, jusqu'alors confiée aux acteurs étatiques répressifs 
(police et justice). Ces passeurs, détenteurs de ressources biographiques et institutionnelles leur permettant de diffuser leurs croyances centrales et de se mouvoir dans les espaces institutionnels, œuvrent au recadrage d'une politique publique déjà existante. La circulation des normes de ces passeurs connaît, dans un premier temps, des résistances face aux acteurs détenteurs d'un savoir traditionnel sans que celles-ci ne soient pourtant durables puisque leurs idées se diffusent et s'institutionnalisent. Cela s'explique par le développement d'une seconde génération de passeurs engagés de la société civile, mus par des convictions personnelles et anticipant des rétributions, qui permettent la consolidation d'un réseau d'acteurs promouvant une démocratisation de la lutte contre l'extrémisme de droite.

Enfin, J. Geeraert étudie les passeurs de normes du soin de la précarité, en prenant l'exemple d'une petite structure d'accès aux soins pour les personnes éloignées du système de santé (PASS). Sa contribution montre l'évolution du profil sociologique des passeurs selon la temporalité du transfert. Dans la première phase d'importation (1990-1998), le transfert fait intervenir des médecins au fort capital social, occupant des positions dominantes au sein de leur hôpital et caractérisés par une double appartenance au champ de la médecine humanitaire et à celui de la médecine hospitalière. La seconde phase (2000-2015) est une phase de consolidation, de défense et de traduction des normes propres à la PASS dans l'institution hospitalière. Elle met en scène des acteurs devenus des professionnels des PASS mais qui, pour autant, sont toujours contraints de transférer des normes tant l'institution qu'ils défendent est fragile. Aussi, ces passeurs de la seconde phase cherchent à consolider le transfert selon deux modes : par la défense et l'ancrage des normes telles qu'elles ont été léguées par les premiers passeurs; par l'adaptation des PASS aux normes néomanagériales désormais dominantes à l'hôpital. Ces passeurs de deuxième génération se distinguent entre eux selon leurs stratégies (individuelles ou collectives) et leurs arènes (ministère, agence régionale de santé).

Fabien Jobard est directeur de recherches au CNRS, au Centre de recherches sociologiques sur le droit et les institutions pénales. Il dirige par ailleurs le Groupement européen sur les normativités (GERN). Ses travaux portent sur la police et l'ordre public, ainsi que sur la justice, la perception du crime et de la peine dans une perspective comparée (CESDIP, 43 bd Vauban, 78280 Guyancourt, fabjob@ cesdip.fr) 